\title{
Depressive Symptoms and Career-Related Goal Appraisals: Genetic and Environmental Correlations and Interactions
}

\author{
Katariina Salmela-Aro, ${ }^{1,2}$ Sanna Read, ${ }^{1,2}$ Eero Vuoksimaa,, ${ }^{3,4}$ Tellervo Korhonen, ${ }^{3}$ Danielle M. Dick, ${ }^{5}$ \\ Jaakko Kaprio, ${ }^{3,7,8}$ and Richard. J. Rose ${ }^{6}$ \\ ${ }^{1}$ University of Jyväskylä, Jyväskylä, Finland \\ ${ }^{2}$ Collegium for Advanced Studies, University of Helsinki, Helsinki, Finland \\ ${ }^{3}$ Department of Public Health, Hjelt Institute, University of Helsinki, Helsinki, Finland \\ ${ }^{4}$ Department of Psychiatry \& Center for Behavioral Genomics, Twin Research Laboratory, University of California, San \\ Diego, La Jolla, CA, USA \\ ${ }^{5}$ Virginia Institute for Psychiatric and Behavioral Genetics, Department of Psychiatry, Virginia Commonwealth University, \\ Richmond, VA, USA \\ ${ }^{6}$ Department of Psychological and Brain Sciences, Indiana University, Bloomington, IN, USA \\ ${ }^{7}$ National Institute for Health and Welfare, Department of Mental Health and Substance Abuse Services, Helsinki, Finland \\ ${ }^{8}$ Institute for Molecular Medicine, University of Helsinki, Helsinki, Finland
}

\begin{abstract}
In order to further understand why depressive symptoms are associated with negative goal appraisals, the present study examined the genetic and environmental correlations and interactions between depressive symptoms and career-related goal appraisals. A total of 1,240 Finnish twins aged 21-26 years completed a questionnaire containing items on the appraisal of their career goals along five dimensions: importance, progress, effort, strain, and self-efficacy. In the same questionnaire, the 10-item General Behavior Inventory assessed depressive symptoms. Structural equation modeling was used to evaluate the genetic and environmental correlations and gene-environment interactions between the career-goal appraisals and depressive symptoms. Associations were identified, and were attributed to environmental factors. Of the career-related goal appraisals, the shared environmental component was of a higher magnitude for the dimension of strain among the depressed compared with non-depressed subjects. The results indicate that the interplay between depressive symptoms and negative career-related goal appraisals is significantly affected by environmental factors, and thus possibly susceptible to targeted interventions.
\end{abstract}

Keywords: depressive symptoms, career-related goal appraisals, genetic correlation, gene-environment interaction, twin, young adults

Career-related goals are highly important in a successful transition to adulthood at the stage when young people face the move from education to employment. Mental health and career development are intertwined in young adulthood, and depressive symptoms are more prevalent at this age than in early adolescence (Adkins et al., 2009). Some recent evidence shows that negative career-goal appraisals are associated with reduced well-being, such as a higher level of depressive symptoms (e.g., Maier \& Brunstein, 2001). However, the origin of this relationship is not clear. To shed light on the underlying factors, this study investigated possible genetic and environmental factors that are common to negative career-goal appraisals and depressive symptoms among young adults. Further, we explored whether the rel- ative contribution of genetic and environmental effects in career-goal appraisals differ as a function of depression. Understanding the origin of the associations can be useful in developing targeted interventions to improve well-being in young adulthood.

The association between depressive symptoms and motivational systems, such as personal goals, is discussed in several theoretical approaches (Salmela-Aro \& Nurmi, 1996).

RECEIVED 5 March 2014; ACCEPTED 12 May 2014. First published online 16 June 2014.

ADDRESS FOR CORRESPONDENCE: Katariina Salmela-Aro, Collegium for Advanced Studies, University of Helsinki, Helsinki, Finland. E-mail: katariina.salmela-aro@helsinki.fi 
According to cognitive theories of depression, underlying dysfunctional cognitions about the self and the external world can lead to depression when negative life events are encountered. Self-regulation theories posit that maladaptive goal appraisals increase vulnerability because they can disrupt the process of pursuing and attaining valued personal goals. A number of studies have investigated the cross-sectional association between depression and goal appraisals (Meyer et al., 2004), and more specifically between depression and work-goal appraisals (e.g., Pomaki et al., 2004). These studies reported weak associations. Moreover, the associations between goal appraisals and emotional well-being have been found to be stronger among those who express a stronger commitment to their goals than among those who express a weaker commitment (Maier \& Brunstein, 2001). In a longitudinal study, Salmela-Aro and Nurmi (1996) found that depressive symptoms were likely to precede negative goal appraisals. Pomaki et al. (2006) concluded that poor goal self-efficacy tended to increase depressive symptoms, which in turn could further intensify goal-related negative emotions. However, the origins of the individual differences are not clear. Are there shared genetic and environmental factors that underlie both depressive symptoms and negative career-goal appraisals? Are the genetic and environmental factors in career-goal appraisals dependent in magnitude on the level on depressive symptoms? This study investigates these questions using a sample of young adult twins.

Twin studies of depressive symptoms have generally shown a rather modest genetic impact and a considerable environmental impact in explaining individual differences in depressive symptoms in adulthood (e.g., Jang et al., 1996). Environmental influences on depression are attributable to factors unique to each individual family member whereas the effect of shared environmental factors in families on adult depressive mood is negligible. A moderate genetic influence has been found in major depression (Kendler \& Myers, 2010). It has been suggested that the genetic effects of depressive symptoms may be indirect and expressed via gene and environment correlations and interactions (Rice, 2009). Thus, it is important to study differences in genetic and environmental associations across different levels of depression.

Much research has been conducted on the role of genetic and environmental factors in depressive symptoms, yet little is known about the origins of variation in careergoal appraisals. Some studies, however, have focused on related motivational characteristics. The results show moderate heritability in personal goals (Salmela-Aro et al., 2012). Generally, behavioral characteristics show at least a moderate genetic influence, varying between $25 \%$ and $60 \%$, depending on the characteristic in question, while depression shares genetic effects with some personality traits, particularly neuroticism (e.g., Kendler \& Myers, 2010), which may represent negative self-concerns similar to negative careergoal appraisals.
The interplay between depressive symptoms and careergoal appraisals may not be uniform across individuals. Heritability estimates of appraisals may depend on the level of depressive symptoms, referring to gene-environment interaction (GE interaction). According to the diathesisstress model, sensitivity to environmental risk factors is stronger in individuals who are at genetic risk for depression (Plomin \& Rutter, 1998). The model predicts more extensive genetic effects in less advantageous environmental circumstances. Bronfenbrenner and Ceci (1994) adopt an alternative approach in their bio-ecological model: genetic potential is better actualized when proximal processes that is, processes that enhance developmental functioning - are present. Furthermore, the mechanism behind GE interaction may be a control process in which restrictive environmental conditions reduce the role of genetic influences, the result being that many individuals exhibit the same phenotype/behavior irrespective of their genetic disposition. In general, genetic effects tend to be higher in less restrictive and less stressful environmental contexts (see review, Johnson, 2007). Data on twins and extended kin have shown that the relative proportion of genetic and environmental influences may change depending on contextual factors. For instance, a higher number of negative life events have been associated with higher heritability estimates for depression and anxiety among adolescent girls (Silberg et al., 2001), supporting the diathesis-stress model. Another study revealed the opposite: A decrease in genetic risk for depressive symptoms was observed along with increases in the levels of negative life events and maternal punitive behavior (Lau \& Eley, 2008). Similarly, academic achievement and engagement, peer affiliations, and interpersonal relationships between child and parent have been found to modify genetic and environmental contributions to internalizing disorders (Hicks et al., 2009), indicating lower heritability when an adverse context is present.

The present study investigated the following three research questions.

1. To what extent is the association between depressive symptoms and negative career-goal appraisal attributable to common genetic and/or environmental factors?

2. Is a greater attribution of importance to career goals associated with a stronger association between depressive symptoms and negative career-goal appraisal?

3. Is a higher level of depressive symptoms associated with higher heritability, or with stronger environmental influences on negative career-goal appraisal?

\section{Method}

\section{Sample}

Data from the FinnTwin12 study were used. The subjects of this population-based longitudinal study comprise five consecutive and complete twin cohorts born between 1983 
and 1987. At the initial stage, baseline questionnaires were sent to all twins and their parents when the twins were 1112 years old. A total of 2,724 families returned the questionnaires (participation rate 87\%). Of these, a subsample of 1,035 families was invited to participate in a more intensive study protocol when the twins were 12 and 14 (Kaprio et al., 2002). This same subsample was invited to participate in the fourth wave of data collection in 2005-2009, when the twins were 21-26 years old (the third wave of data collection was a questionnaire study when the twins were 17 years old). All the twins who participated in the fourth wave of data collection were contacted for this study, yielding a response rate of $87 \%$; for a more detailed description see Kaprio et al. (2002) and Kaprio (2013). The focus in the present study was on personal goals and depressive symptoms, and hence individuals who did not respond to the relevant items in the questionnaire $(n=89)$ were excluded from the analysis. In total, 1,240 individuals were included.

The zygosity of the twins was determined by means of a validated questionnaire on physical similarity (Sarna et al., 1978), with added questions for the younger twins. In addition, DNA was used to determine the zygosity of the 397 same-sex pairs, and in $97 \%$ of the cases confirmed the questionnaire-based assessment. The sample used in this study thus comprised 135 monozygotic female (MZF) twin pairs, 101 monozygotic male (MZM) twin pairs, 96 dizygotic female (DZF) twin pairs, 73 dizygotic male (DZM) twin pairs, and 150 dizygotic opposite-sex (DZOS) twin pairs. In addition, there were 130 individuals whose cotwin did not participate in the study. In these cases the individual twin contributed to the calculation of means and variances/prevalences.

\section{Measures}

Career goals. The twins filled out a questionnaire on personal goals (Salmela-Aro \& Nurmi, 1996). They were then asked to name a career goal and to assess it on 11 appraisal items rated on a 7-point scale. The sum scores of the five dimensions of career-goal appraisals were as follows: importance ('How important is your career goal?' $\alpha=0.79$ ); progress ('How far have you progressed in reaching this career goal?' $\alpha=0.62$ ); effort ('How much time and effort have you invested in this career goal?' $\alpha=0.89$ ); strain ('How stressful is your career goal?' $\alpha=0.85$ ); and self-efficacy ('To what extent do you know what to do to reach this career goal?' $\alpha=0.76$ ).

Depressive symptoms were assessed using the General Behavior Inventory (GBI) developed by Depue (1987). In this study, a shortened version of the depression subscale was used based on a reliability analysis with another Finnish sample (Kokko \& Pulkkinen, 1998). It contained 10 items which were answered on a 4-point scale from $0=$ never to $3=$ very often. The sum score of the scale was calculated $(\alpha=0.90)$.
Since female gender, higher age, and lower educational status have been found to be associated with depressive symptoms and negative career-goal appraisals (Nurmi et al., 2002), the models were adjusted for gender, age in years, currently studying, including part-time studying versus not studying, and qualifications dichotomized into having and not having a matriculation diploma. The age range was 2126 years, with a mean age of 22.4 and standard deviation $(S D)$ of 0.70 . The majority of men and women were currently studying at least part-time (51\%, 59\%), and $54 \%$ of the men and $70 \%$ of the women had a high school matriculation diploma.

\section{Analysis}

The career-goal appraisals were the dependent variables in the analysis. The phenotypic associations between careergoal appraisal and depressive symptoms were analyzed using linear regression models with Generalized Estimation Equations (GEE) that take into account the dependency between the members of the twin pairs. The analyses were carried out separately for men and women. Age, current studying status, and qualification were adjusted in the models. In the next step, the importance of the career goal was taken into account in the association between the other dimensions of career-goal appraisals and depressive symptoms. The interaction terms importance and depressive symptoms were added to test whether the association between goal appraisals and depressive symptoms was stronger - the higher the importance attributed to the career goal.

Individual variation in the variables was decomposed into additive genetic (A), common environment (C), and unique environment (E) effects. A was constrained to be 1 for the monozygotic (MZ) twin pairs and 0.5 for the dizygotic (DZ) twin pairs. $C$ was constrained to be 1.0 in both $\mathrm{MZ}$ and $\mathrm{DZ}$ pairs. E refers to all the environmental effects that make members of a twin pair different from each other and is assumed to contain uncorrelated measurement error.

Within-pair intraclass correlations were calculated for the MZF and MZM pairs, the DZF and DZM pairs, and the DZOS pairs. Intraclass correlations in MZ pairs that are twice or more as larger as in DZ pairs are indicative of additive genetic influence in the trait and the possible presence of genetic dominance, whereas if the MZ twin correlations are less than twice the $\mathrm{DZ}$ correlations, a shared environmental effect is likely to be present. The equality of means and variances between the $\mathrm{MZ}$ and $\mathrm{DZ}$ twins was tested in a saturated model in which the means and variances are not constrained to be equal across the twin groups; this is a basic assumption of the twin model to ensure that twins of both zygosities are from the same base population.

The next step was to fit univariate models to estimate genetic and environmental components to the career-related goal appraisals. Raw data, that is, data from all subjects, 
TABLE 1

Correlations, Means, and Variances for Career-Goal Appraisals and Depressive Symptoms

\begin{tabular}{|c|c|c|c|c|c|c|c|}
\hline & Importance & Progress & Effort & Strain ${ }^{a}$ & Self-efficacy & Depressive symptoms & Mean (SD) \\
\hline \multicolumn{8}{|l|}{ Career-goal appraisals } \\
\hline Importance & - & $0.54^{* * *}$ & $0.59^{* * *}$ & $0.30^{* * *}$ & $0.53^{* * *}$ & $-0.15^{* * *}$ & 5.74 (1.09) \\
\hline Progress & $0.45^{* * *}$ & - & $0.67^{* * * *}$ & $0.13^{* * *}$ & $0.62^{* * *}$ & $-0.25^{* * *}$ & 5.18 (1.18) \\
\hline Effort & $0.52^{* * * *}$ & $0.59^{* * *}$ & - & $0.29^{* * *}$ & $0.44^{* * *}$ & $-0.17^{* * *}$ & $4.73(1.37)$ \\
\hline Strain $^{a}$ & $0.29 * * *$ & $0.11^{* *}$ & $0.32^{* * *}$ & - & $0.16^{* * *}$ & $-0.12^{* *}$ & $4.36(1.54)$ \\
\hline Self-efficacy & $0.37^{* * * *}$ & $0.57^{* * *}$ & $0.35^{* * *}$ & -0.02 & - & $-0.22^{* * *}$ & $5.70(0.98)$ \\
\hline Depressive symptoms & -0.07 & $-0.18^{* * *}$ & $-0.09^{*}$ & $-0.12^{* *}$ & $-0.23^{* * *}$ & - & 13.60 (4.09) \\
\hline Mean $(S D)$ & $6.03(0.88)$ & $5.15(1.17)$ & $4.83(1.46)$ & $4.79(1.55)$ & $5.67(0.91)$ & $15.32(4.94)$ & - \\
\hline
\end{tabular}

Note: Men $(n=569)$ above the diagonal, women $(n=671)$ below the diagonal.

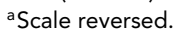

**** $p<.001,{ }^{* *} p<.01, * p<.05$.

were used. Possible effects of age, gender, working status, and having a matriculation diploma were adjusted using residuals from the regressions. Based on the patterns in the intraclass correlation, the best fitting full model comprised the $\mathrm{A}, \mathrm{C}$, and $\mathrm{E}$ components. The restricted models tested whether the A or the C component, or both, could be dropped. The difference between the full models and the restricted models, that is, the difference in $-2 \log$ likelihood $(-2 L L)$, is distributed as $\chi^{2}$, the degrees of freedom being the difference in the number of parameters that are estimated. A significant $\chi^{2}$ difference indicates that reduction in the model significantly reduces its fit to the data. The models were also compared according to Akaike's Information Criterion (AIC): a smaller value indicates a better model.

Inter-trait correlations were calculated to examine associations between depressive symptoms and the career-goal appraisals. These calculations were carried out both within the individual (cross-trait within-twin correlations for assessing phenotypic correlations) and between the members of twin pairs (cross-trait within-pair correlations for assessing genetic correlations between the traits). The bivariate Cholesky decomposition was used to estimate genetic and environmental associations between depressive symptoms and career-goal appraisals. Depressive symptoms and career-goal appraisals were treated as continuous in the model. Because depressive symptoms were skewed, a Maximum Likelihood estimator (MLR) with robust standard errors for non-normal distributions was used. The significance of the terms in the model was estimated by calculating the $95 \%$ confidence intervals $(95 \% \mathrm{CI})$.

Gene-environment interaction was analyzed by fitting a model that included parameters for A, C, and E that were independent of the moderator effect and those that depended on the moderator $\left(a^{\prime}, c^{\prime}\right.$, and $\left.e^{\prime}\right)$. There was no indication of the presence of moderation on the covariance between depressive symptoms and career-goal appraisals when the full bivariate model as suggested by Purcell (2002) was fitted. Because of this, we used the extended univariate moderator model (van der Sluis et al., 2012), which is more powerful in this case. Following the extended univariate moderator model by van der Sluis et al. (2012), the tested models were adjusted for moderator effects on the means within each cotwin and between the twins in a pair. The moderator effects on means were allowed to vary between $\mathrm{MZ}$ and $\mathrm{DZ}$ twins. After the full model, a number of restricted models were tested, in which the moderator effects were fixed at zero. A significant $\chi^{2}$ difference test indicated that the component was necessary.

\section{Results}

The means, variances, and correlations are shown in Table 1. Table 2 shows the effect of depressive symptoms on careergoal appraisals when age, matriculation, and studying status were controlled for. Depressive symptoms were associated with more negative career-goal appraisals. In order to find out whether the association was stronger among those who rated their career goal more highly, we tested the interaction terms between goal importance and depressive symptoms regressed on career goals. Importance of career goal did not modify the association between depressive symptoms and career goals (results not shown).

Table 3 shows the within-pair correlations for twins. The MZ correlations were modest and about twice the level of the $\mathrm{DZ}$ correlations, indicating that there may be a modest genetic influence on career-goal appraisals.

The univariate models for the career-goal appraisals and depressive symptoms showed that in all cases the shared environmental effect could be dropped without weakening the fit (the results not shown). The additive genetic component of the career-goal appraisals of progress and effort was non-significant in the nested models compared with the full model. However, dropping both A and C worsened the fit significantly, indicating that one of the components should be retained. The AIC indices showed that the AE model fitted better than the CE model. The best fitting models for the career-goal appraisals and depressive symptoms included the additive genetic and non-shared environmental components. Overall, the estimates for the A components were modest, except in the case of depressive symptoms, where it was high. 
TABLE 2

Association Between Career-Goal Appraisals and Depressive Symptoms

\begin{tabular}{|c|c|c|}
\hline & $\begin{array}{l}\text { Men }(n=569) \\
F\end{array}$ & $\begin{array}{l}\text { Women }(n=671) \\
F\end{array}$ \\
\hline \multicolumn{3}{|l|}{ Importance } \\
\hline Depressive symptoms & $10.34^{* *}$ & 0.97 \\
\hline Age & 0.64 & 0.01 \\
\hline Studying (no vs. yes) & 2.51 & $18.60^{* * *}$ \\
\hline Qualification (matriculation diploma vs. no) & $7.64^{* *}$ & 1.64 \\
\hline \multicolumn{3}{|l|}{ Progress } \\
\hline Depressive symptoms & $34.68^{* * *}$ & $14.03^{* * *}$ \\
\hline Age & 3.35 & 2.20 \\
\hline Studying (no vs. yes) & $9.60^{* *}$ & $30.98^{* * *}$ \\
\hline Qualification (matriculation diploma vs. no) & 2.00 & 2.63 \\
\hline \multicolumn{3}{|l|}{ Effort } \\
\hline Depressive symptoms & $15.78^{* * *}$ & 2.31 \\
\hline Age & 0.04 & 2.00 \\
\hline Studying (no vs. yes) & $5.48^{*}$ & $12.35^{* * *}$ \\
\hline Qualification (matriculation diploma vs. no) & 0.02 & 3.25 \\
\hline \multicolumn{3}{|l|}{ Strain ${ }^{a}$} \\
\hline Depressive symptoms & $14.41^{* * *}$ & $19.05^{* * *}$ \\
\hline Age & 0.38 & 0.33 \\
\hline Studying (no vs. yes) & $6.37^{*}$ & $14.96^{* * *}$ \\
\hline Qualification (matriculation diploma vs. no) & $20.20^{* * *}$ & $20.43^{* * *}$ \\
\hline \multicolumn{3}{|l|}{ Self-efficacy } \\
\hline Depressive symptoms & $22.43^{* * *}$ & $32.53^{* * *}$ \\
\hline Age & 0.00 & 0.02 \\
\hline Studying (no vs. yes) & 2.42 & 3.76 \\
\hline Qualification (matriculation diploma vs. no) & $12.84^{* * *}$ & 0.14 \\
\hline
\end{tabular}

Note: Standard errors adjusted for the dependency between the members of twin pairs.

a Scale reversed

${ }^{* * *} p<.001,{ }^{* *} p<.01, * p<.05$.

TABLE 3

Within-Pair Correlations $(95 \% \mathrm{Cl})$ for Career-Goal Appraisals and Depressive Symptoms

\begin{tabular}{lcc} 
& $\mathrm{MZ}$ & \\
& $\mathrm{n}$ of pairs $=236$ & $\mathrm{DZ}$ \\
$\mathrm{n}$ of pairs $=319$ \\
\hline Goal appraisals & & \\
Importance & 0.24 & 0.07 \\
& $(0.12-0.36)$ & $(0-0.19)$ \\
Progress & 0.16 & 0.04 \\
& $(0.01-0.30)$ & $(0-0.16)$ \\
Effort & 0.18 & 0.01 \\
& $(0.15-0.31)$ & $(0-0.07)$ \\
Strain & 0.27 & 0.07 \\
& $(0.14-0.39)$ & $(0-0.17)$ \\
Self-efficacy & 0.18 & 0.03 \\
& $(0.04-0.30)$ & $(0-0.08)$ \\
Depressive symptoms & 0.51 & 0.19 \\
& $(0.35-0.66$ & $(0.06-0.31)$ \\
\hline
\end{tabular}

Note: $M Z=$ Monozygotic female twins, $D Z=$ Dizygotic twins.

Table 4 shows the genetic and environmental effects that were specific for and shared between depressive symptoms and the career-goal appraisals. The genetic effects remained specific to depressive symptoms and the goal appraisals, the shared genetic effects (A common) being very low and nonsignificant. The phenotypic correlations between depressive symptoms and the career-goal appraisals are attributable to shared unique environmental factors (E common): the non-shared environmental effects of depressive symptoms overlapped $13 \%, 20 \%$, and $24 \%$ of the non-shared en- vironmental effects of progress, effort, and self-efficacy respectively.

The GE interaction models in Table 5 show that most of the moderation terms could be removed without worsening the model fit. The only significant finding, apart from the slight moderation on the non-shared environmental components and the moderation on the means, was that related to the appraisal of career-goal strain; there was a moderation effect on environmental effects $\mathrm{C}$ and E. Figure 1 shows the change by the level of depressive symptoms. The additive genetic effect on strain remained constant with increase in depressive symptoms. The proportion of shared environmental effects increased, and unique environmental effects decreased, along with higher scores for depressive symptoms.

\section{Discussion}

This is the first study to examine the possible genetic and environmental factors that are common to negative careergoal appraisals and depressive symptoms among young adults. The results highlight the role of environmental factors in the associations between depressive symptoms and career-goal appraisals. A number of possible explanations for the findings, and their implications, are discussed.

First, the phenotypic associations were relatively low, although in pattern and magnitude they resembled previous findings on specific career-goal appraisals. The modest 
TABLE 4

Percentages of Common and Trait-Specific Additive (A) and Non-Shared Environmental (E) Effects for Career-Goal Appraisals and Depressive Symptoms

\begin{tabular}{|c|c|c|c|c|}
\hline & A common\% $(95 \% \mathrm{Cl})$ & E common $\%(95 \% \mathrm{Cl})$ & A specific\% $(95 \% \mathrm{Cl})$ & E specific\% $(95 \% \mathrm{Cl})$ \\
\hline Depressive symptoms with & - & - & $\begin{array}{l}50 \\
(33-68)\end{array}$ & $\begin{array}{l}50 \\
(34-65)\end{array}$ \\
\hline Importance & $\begin{array}{l}6 \\
(0-12)\end{array}$ & $\begin{array}{l}1 \\
(0-7)\end{array}$ & $\begin{array}{l}18 \\
(12-24)\end{array}$ & $\begin{array}{l}75 \\
(68-81)\end{array}$ \\
\hline Progress & $\begin{array}{l}3 \\
(0-11)\end{array}$ & $\begin{array}{l}12 \\
(5-20)\end{array}$ & $\begin{array}{l}16 \\
(8-24)\end{array}$ & $\begin{array}{l}69 \\
(61-77)\end{array}$ \\
\hline Effort & $\begin{array}{l}1 \\
(0-11)\end{array}$ & $\begin{array}{l}20 \\
(10-30)\end{array}$ & $\begin{array}{l}13 \\
(3-23)\end{array}$ & $\begin{array}{l}66 \\
(56-77)\end{array}$ \\
\hline Strain & $\begin{array}{l}8 \\
(0-18)\end{array}$ & $\begin{array}{l}2 \\
(0-12)\end{array}$ & $\begin{array}{l}23 \\
(14-34)\end{array}$ & $\begin{array}{l}67 \\
(56-76)\end{array}$ \\
\hline Self-efficacy & $\begin{array}{l}2 \\
(0-7)\end{array}$ & $\begin{array}{l}24 \\
(19-29)\end{array}$ & $\begin{array}{l}14 \\
(9-19)\end{array}$ & $\begin{array}{l}60 \\
(55-65)\end{array}$ \\
\hline
\end{tabular}

Note: Based on the bivariate Cholesky decomposition where depressive symptoms are the first and the work-goal appraisal the second variable. Percentages in bold; $95 \%$ confidence intervals in parentheses.

\section{TABLE 5}

Interaction Model of Career-Goal Appraisals With Depressive Symptoms as a Moderator

\begin{tabular}{|c|c|c|c|c|}
\hline & $-2 L L$ & $n$ of parameters & Model comparison & Difference test \\
\hline \multicolumn{5}{|l|}{ Importance } \\
\hline 1. Full model & 2199.24 & 12 & - & - \\
\hline 2. Drop moderation in $A$ & 2199.93 & 11 & 2 vs. 1 & $0.69(1) \mathrm{ns}$ \\
\hline 3. Drop moderation in $C$ & 2199.94 & 10 & 3 vs. 2 & 0.01 (1) ns \\
\hline 4. Drop moderation in $E$ & 2204.59 & 9 & 4 vs. 3 & $4.65(1)^{*}$ \\
\hline $\begin{array}{l}\text { 5. Drop moderation means } \\
\text { Progress }\end{array}$ & 2217.02 & 6 & 5 vs. 3 & $17.08(4)^{* *}$ \\
\hline 1. Full model & 2493.98 & 12 & - & - \\
\hline 2. Drop moderation in $A$ & 2494.28 & 11 & 2 vs. 1 & $0.30(1) \mathrm{ns}$ \\
\hline 3. Drop moderation in $C$ & 2495.22 & 10 & 3 vs. 2 & $0.94(1) \mathrm{ns}$ \\
\hline 4. Drop moderation in $\mathrm{E}$ & 2498.86 & 9 & 4 vs. 3 & 3.64 (1) ns \\
\hline $\begin{array}{l}\text { 5. Drop moderation means } \\
\text { Effort }\end{array}$ & 2530.92 & 5 & 5 vs. 4 & $32.06(4)^{* * *}$ \\
\hline 1. Full model & 2855.99 & 12 & - & - \\
\hline 2. Drop moderation in $A$ & 2857.29 & 11 & 2 vs. 1 & $1.30(1) \mathrm{ns}$ \\
\hline 3. Drop moderation in $\mathrm{C}$ & 2857.38 & 10 & 3 vs. 2 & 0.09 (1) ns \\
\hline 4. Drop moderation in $\mathrm{E}$ & 2858.82 & 9 & 4 vs. 3 & $1.44(1) \mathrm{ns}$ \\
\hline $\begin{array}{l}\text { 5. Drop moderation means } \\
\text { Strain }\end{array}$ & 2878.45 & 5 & 5 vs. 4 & $19.63(4)^{* * *}$ \\
\hline 1. Full model & 2967.01 & 12 & - & - \\
\hline 2. Drop moderation in $A$ & 2967.02 & 11 & 2 vs. 1 & $0.01(1)$ \\
\hline 3. Drop moderation in $C$ & 2971.84 & 10 & 3 vs. 2 & $4.82(1)^{*}$ \\
\hline 4. Drop moderation in $E$ & 2971.58 & 10 & 4 vs. 2 & $4.56(1)^{*}$ \\
\hline $\begin{array}{l}\text { 5. Drop moderation means } \\
\text { Self-efficacy }\end{array}$ & 2980.04 & 7 & 5 vs. 2 & $13.02(4)^{*}$ \\
\hline 1. Full model & 2555.14 & 12 & - & - \\
\hline 2. Drop moderation in $A$ & 2555.95 & 11 & 2 vs. 1 & $0.81(1) \mathrm{ns}$ \\
\hline 3. Drop moderation in $C$ & 2557.10 & 10 & 3 vs. 2 & 1.15 (1) ns \\
\hline 4. Drop moderation in $E$ & 2566.10 & 9 & 4 vs. 3 & $9.00(1)^{* *}$ \\
\hline 5. Drop moderation means & 2597.31 & 5 & 5 vs. 3 & $40.21(4)^{* * *}$ \\
\hline
\end{tabular}

Note: ${ }^{* * *} p<.001,{ }^{* *} p<.01,{ }^{*} p<.05 ;$ ns $=$ non-significant.

correlations suggest that these concepts are relatively independent. Although studying status was taken into account in the analysis, there may continue to be wide contextual differences in this age group in relation to the stage of starting a career. One of the factors we took into account was the importance of the career-related goal. Previous studies have indicated that higher levels of career-goal commitment and importance are associated with a stronger link between wellbeing and positive goal appraisals and, on the other hand, between depression and negative goal appraisals (Maier \& Brunstein, 2001). Few indications of the effect of goal importance on the associations of other goal appraisal dimen- sions with depressive symptoms were found. The different result may be related to the fact that the participants were quite young adults, as the majority of them still studying in tertiary education. They were therefore at a very early stage in their careers when the importance of their career goal may reflect different factors than later in adulthood. Moreover, the overwhelming majority rated their career goal as at least moderately important, resulting in a skewed distribution. This indicates the high importance of the goal at this age stage, the variable possibly suffering from a ceiling effect. The associations tended to be stronger among men. Both educational status and current studying status 


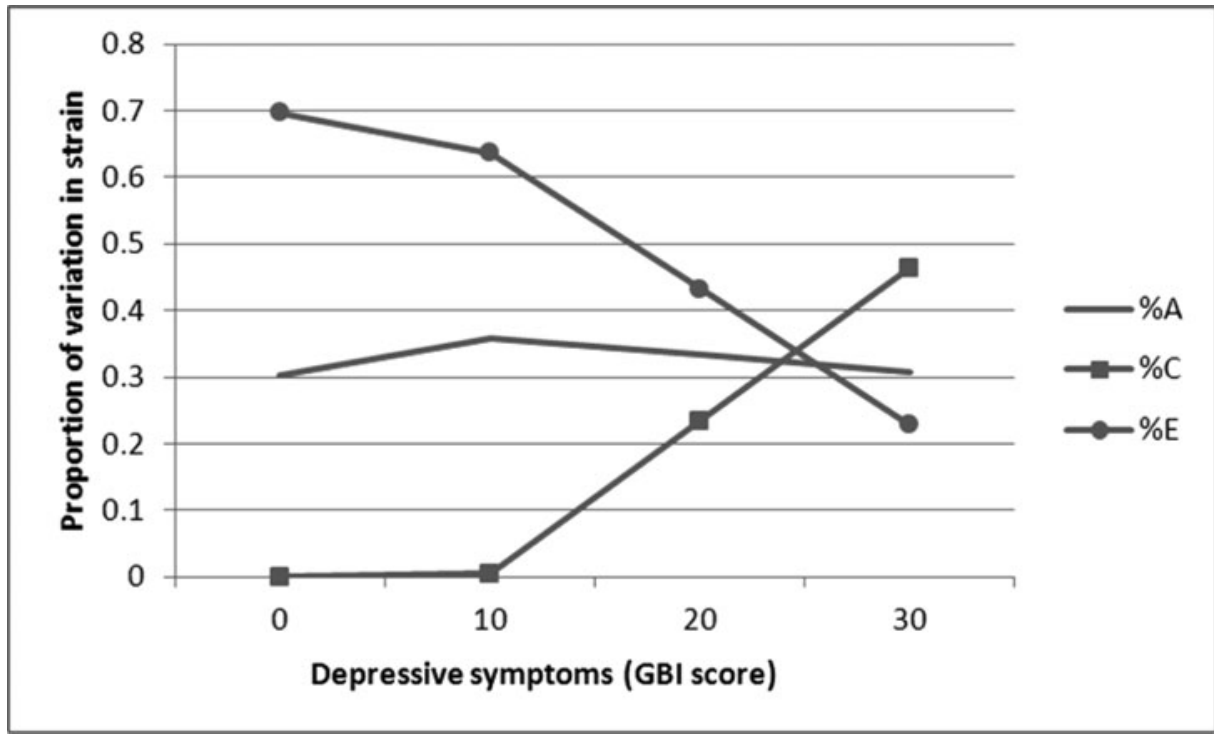

\section{FIGURE 1.}

The proportion of additive genetic $(\% \mathrm{~A})$, shared $(\% \mathrm{C})$, and unique $(\% \mathrm{C})$ environmental effects on strain by the level of depressive symptoms.

were associated with career-goal appraisals, supporting the results of earlier studies (Salmela-Aro \& Nurmi, 1996).

The heritability estimates for career-goal appraisals were modest whereas that for depressive symptoms were higher. On the evidence of previous studies on goals, it would be reasonable to expect that a motivational measure, such as goal appraisal, would exhibit a rather lower level of genetic influence (Salmela-Aro et al., 2012), whereas depressive symptoms may show at least a moderate genetic effect. However, the confidence interval was wide, indicating that the range of heritability could be anything from moderate to high.

Given the modest heritability, we were unlikely to find a high proportion of the total variance to be due to shared genetic association between negative career-goal appraisals and depressive symptoms. It is important to note that when heritability and phenotypic correlation are low, the genetic correlation can still be high. This would indicate that most genetic factors are shared, but these factors do not explain much of the variance of the two traits. The results did not reveal any shared environmental effects in either case, suggesting that the associations are attributable to genetic or non-shared environmental factors. The origin of common variation between progress, effort, and self-efficacy in career goals and depressive symptoms was attributable to nonshared environmental factors. The shared genetic components were minimal, and turned out to be non-significant: Most of the individual variations in career-goal appraisals and depressive symptoms seem to be unique to the individual, and the small proportion that is shared seems generally attributable to environmental effects. Thus, career goals are a suitable focus of intervention (Salmela-Aro et al., 2012).
It is also possible that no overall associations were found because of the underlying interactions. It has been suggested that the genetic effects of depressive symptoms may be indirect and work via gene and environment correlations and interactions (Rice, 2009). We found little evidence of a changing genetic contribution to career-goal appraisal, depending on the level of depression. In light of this result, it is unlikely that the overall associations remained low because of the presence of GE interaction. Of the five goal-appraisal dimensions, three (importance, progress, effort) showed no GE interaction. For self-efficacy, moderation was found only on the non-shared environmental component, which may be due to sample size, meaning that the effects were not strong enough to be detected. In the case of goal strain, the magnitude of the genetic effects remained constant whereas the shared environmental effect grew bigger and the unique environmental effect smaller among those showing a high level of depressive symptoms. Previous studies attempting to disentangle the possible GE interactions related to depression and motivational factors are very few, which makes it difficult to paint a full picture. Moreover, studies focusing on GE interactions with depression in different adverse life events related to specific genes have reported mixed findings and often fail to replicate the results of previous research. One reason why the literature contains very few studies on the genetic associations between depressive symptoms and the motivational system may be that research results remain unpublished because only non-significant associations are found. Finding non-significant or non-genetic associations is nevertheless important information and should be taken seriously so as to cover the whole spectrum of the issue in question and not only the skewed tail of a distribution. 
There are several reasons why very few GE interactions were found, and it may be that a range of contextual factors contribute in various ways to career-goal appraisal. We only assessed the level of depression at one point in time, a choice which may have influenced the results. Multiple testing could also influence the results, and therefore replications using other samples are important. Similarly, previous research on depression focuses on negative factors. The diathesis-stress model and bio-ecological model approaches GE interaction from different perspectives. The former model predicts more extensive genetic effects in less advantageous environmental circumstances, whereas the latter expects the heritability to be lower in an adverse environment. It is possible that the two approaches detect different underlying mechanisms. It may be useful in future studies to assess the influence of positive environmental factors on heritability in positive career-goal appraisals.

\section{Acknowledgments}

Data collection and analysis of FinnTwin12 has been supported by the Academy of Finland (141054, 265240, 263278, 264146 to JK) and NIH AA-12502, AA-00145, and AA09203 to RJRose, AA15416 and K02AA018755 to DM Dick, analysis for data SA 273361 for KSA.

\section{References}

Adkins, D. E., Wang, V., Dupre, M. E., van den Oord, E. J. C. G., \& Elder, G. H. (2009). Structure and stress: Trajectories of depressive symptoms across adolescence and young adulthood. Social Forces, 88, 31-60.

Bronfenbrenner, U, \& Ceci, S. J. (1994). Nature-nurture reconceptualized in developmental perspective: A bio-ecological model. Psychological Review, 101, 568-586.

Depue, R. (1987). General behavior inventory. Ithaca, NY: Department of Psychology, Cornell University.

Hicks, B. M., DiRago, A. C., Iacono, W. G., \& McGue, M. (2009). Gene-environment interplay in internalizing disorders: Consistent findings across six environmental risk factors. Journal of Child Psychology and Psychiatry, 50, 13091317.

Jang, K. L., Livesley, W. J., \& Vernon, P. A. (1996). Heritability of the big five personality dimensions and their facets: A twin study. Journal of Personality, 64, 577-591.

Johnson, W. (2007). Genetic and environmental influences on behavior: Capturing all the interplay. Psychological Review, 114, 423-440.

Kaprio, J. (2013). The Finnish Twin Cohort study: An update. Twin Research and Human Genetics, 16, 157-162.

Kaprio, J., Pulkkinen, L., \& Rose, R. J. (2002). Genetic and environmental factors in health-related behaviors: Studies on Finnish twins and twin families. Twin Research, 5, 366371.

Kendler, K. S., \& and Myers, J. (2010).The genetic and environmental relationship between major depression and the five-factor model of personality. Psychological Medicine, 40, 801-806.

Kokko, K., \& Pulkkinen, L. (1998). Unemployment and psychological distress: Mediator effects. Journal of Adult Development, 5, 205-217.

Lau, J. Y. F., \& Eley, T. C. (2008). Disentangling geneenvironment correlations and interactions on adolescent depressive symptoms. Journal of Child Psychology and Psychiatry, 49, 142-150.

Maier, G. W., \& Brunstein, J. C. (2001). The role of personal work goals in newcomers' job satisfaction and organizational commitment: A longitudinal analysis. Journal of Applied Psychology, 86, 1034-1042.

Meyer, B., Beevers, C. G., \& Johnson, S. L. (2004). Goal appraisals and vulnerability to bipolar disorder: A personal project analysis. Cognitive Therapy and Research, 28, 173182.

Nurmi, J., Salmela-Aro, K., \& Koivisto, P. (2002). Goal importance and related achievement beliefs and emotions during the transition from vocational school to work: Antecedents and consequences. Journal of Vocational Behavior, 60(2), 241-261. doi:10.1006/jvbe.2001.1866

Plomin, R., \& Rutter, M. (1998). Child development, molecular genetics, and what to do with genes once they found. Child Development, 69, 1223-1242.

Pomaki, G., Maes, S., \& ter Doest, L. (2004). Work conditions and employees' self-set goals: Goal processes enhance prediction of psychological distress and well-being. Personality and Social Psychology Bulletin, 30, 685-694.

Pomaki, G., ter Doest, L., \& Maes, S. (2006). Goals and depressive symptoms: Cross-lagged effects of cognitive versus emotional goal appraisals. Cognitive Therapy and Research, 30, 499-513.

Purcell, S. (2002). Variance components models for geneenvironment interaction in twin analysis. Twin Research, 5, 554-571.

Rice, F. (2009). The genetics of depression in childhood and adolescence. Current Psychiatry Reports, 11, 167-173.

Salmela-Aro, K., Mutanen, P., \& Vuori, J. (2012). Promoting career preparedness and intrinsic work-goal motivation: RCT intervention. Journal of Vocational Behavior, 80, 6775.

Salmela-Aro, K., \& Nurmi, J.-E. (1996). Depressive symptoms and personal project appraisals: A cross-lagged longitudinal study. Personality and Individual Differences, 21, 373381.

Sarna, S., Kaprio, J., Sistonen, P., \& Koskenvuo, M. (1978). Diagnosis of twin zygosity by mailed questionnaire. Human Heredity, 28, 241-254.

Silberg, J., Rutter, M., Neale, M., \& Eaves, L. (2001). Genetic moderation of environmental risk for depression and anxiety in adolescent girls. British Journal of Psychiatry, 179, 116-121.

van der Sluis, S., Posthuma, D., \& Dolan, C. V. (2012). A note on false positives and power in $\mathrm{G} \times \mathrm{E}$ modelling of twin data. Behavior Genetics, 42, 170-186. 